\title{
Risk Factors for Adverse Pregnancy Outcomes among Zhuang Ethnic Pregnant Women: A Cohort Study in Guangxi, China*
}

\author{
Bao-ying FENG ${ }^{1 \dagger}$, Yang $\mathrm{PENG}^{2 \dagger}$, Jun $\mathrm{LIANG}^{3}$, Li WU1 ${ }^{1}$, Qun-jiao JIANG ${ }^{1}$, Shun LIU ${ }^{3}$, Xiao-yun ZENG ${ }^{3}$, Dong-ping HUANG ${ }^{1}$, \\ Xiao-qiang QIU ${ }^{3}$, Han LI $^{1 \#}$ \\ ${ }^{1}$ Department of Sanitary Chemistry, School of Public Health, Guangxi Medical University, Nanning 530021, China \\ ${ }^{2}$ Department of Environmental and Occupational Health, School of Public Health, Guangxi Medical University, Nanning \\ 530021, China \\ ${ }^{3}$ Department of Epidemiology and Health Statistics, School of Public Health, Guangxi Medical University, Nanning 530021, \\ China
}

(C) The Author(s) 2021

\begin{abstract}
Summary: Risk factors for adverse pregnancy outcomes among Zhuang ethnic pregnant women are unclear. This study analyzed the incidence and risk factors related to preterm birth (PB), low birth weight (LBW) and macrosomia in Zhuang population. We conducted a prospective cohort study of 9965 Zhuang pregnancy women in Guangxi, China. Information on mothers and newborns was obtained by using questionnaires and referring to medical records. Multivariate logistic regression analyses were used to evaluate the association between related factors and adverse pregnancy outcomes. Our results showed that the incidence of PB, LBW and macrosomia in Zhuang people was $5.55 \%, 5.64 \%$ and $2.19 \%$, respectively. Maternal age $\geq 36$ years $(\mathrm{OR}=2.22,95 \%$ CI: 1.51-3.27) was related to a higher incidence of PB. Those with pre-pregnancy body mass index $(\mathrm{BMI})<18.5 \mathrm{~kg} / \mathrm{m}^{2}(\mathrm{OR}=1.91,95 \% \mathrm{CI}: 1.45-2.51)$, and had a female fetus $(\mathrm{OR}=1.74,95 \% \mathrm{CI}$ : $1.36-2.23)$ were more likely to have LBW infants. Maternal age between 31 and 35 years $(\mathrm{OR}=1.76$, 95\% CI: 1.03-2.99) and pre-pregnancy overweight or obesity (OR=1.79, 95\% CI: 1.15-2.80) were associated with a higher risk of macrosomia. The protective factors of macrosomia were maternal pre-pregnancy BMI $<18.5 \mathrm{~kg} / \mathrm{m}^{2}(\mathrm{OR}=0.30,95 \% \mathrm{CI}: 0.15-0.60)$ and female fetus $(\mathrm{OR}=0.41,95 \%$ CI: $0.28-0.59)$. Our study provided a reference for maternal and childcare administration among Zhuang population.
\end{abstract}

Key words: Zhuang ethnic; cohort study; low birth weight; preterm birth; macrosomia; risk factors

Preterm birth (PB), low birth weight (LBW) and macrosomia are common types of adverse pregnancy outcomes. According to an international study of 184 countries, the global incidence of PB was $11.1 \%$ in 2010, and it was $7.1 \%$ in $\mathrm{China}^{[1]}$. PB remains a global problem associated with perinatal morbidity and adverse health outcomes ${ }^{[2-5]}$. It has been reported that the risk factors of $\mathrm{PB}$ mainly include the following: maternal age, parity, maternal nutrition during pregnancy, smoking, and gestational diseases ${ }^{[6-8]}$.

Bao-ying FENG, E-mail: bao971@126.com; Yang PENG, E-mail: pyu098@126.com

${ }^{\dagger}$ These authors contributed equally to the work.

"Corresponding author, E-mail: leehan1988@126.com

${ }^{*}$ This project was supported by grants from the National Natural Science Foundation of China (No. 21906032 and No. 81903285), China Postdoctoral Science Foundation (No. 2020M673556XB), Youth Science Foundation of Guangxi Medical University (No. GXMUYSF201822) and the Guangxi Key Research Program (No. AB17195012).
LBW and macrosomia have been reported to be the risk factors of newborn illness and diseases in adulthood ${ }^{[9-12]}$. One prior study reported that the worldwide prevalence of LBW was $14.6 \%$ in $2015^{[9]}$. Some maternal factors such as maternal age, parity, hypertension, and pre-pregnancy body mass index (BMI) have been shown to be related to the risk of $\mathrm{LBW}^{[13-16]}$. The rate of macrosomia increased over the past several decades in many countries. In China, Shan et al found that the percentages of macrosomia in Beijing increased from $6.6 \%$ in 1996 to $9.5 \%$ in 2000 and declined to $7.0 \%$ in $2010^{[17]}$. While Zhang et al found an overall increase in frequency of macrosomia from $8.9 \%$ in 2010 to $10.1 \%$ in 2013 , in Xi'an, China $^{[18]}$. Factors associated with macrosomia may include but not limited to parity, BMI, maternal age, and fetal sex ${ }^{[19-21]}$.

It has been reported that ethnic differences also have an impact on pregnancy outcomes. Diabelková et al analyzed the differences in pregnancy outcomes between Roma and non-Roma mothers, and found that 
the Roma mothers had a higher risk of giving birth to LBW babies ${ }^{[22]}$. A study from the Xinjiang region in China showed that the incidence of LBW among Uighur, Hazakh and Hui ethnic groups was significantly higher than that of Han ethnic group, while the rate of macrosomia among Uighur was lower than that of $\mathrm{Han}^{[23]}$. China is the largest developing country in the world with 56 ethnic groups. Zhuang is the most populous ethnic minority in China, mostly living in the Guangxi Zhuang Autonomous Region. However, to the best of our knowledge, there were few researches on the incidence and risk factors for adverse pregnancy outcomes in the Zhuang people.

In this study, we investigated and analyzed the incidence and risk factors of three adverse pregnancy outcomes (LBW, PB and macrosomia) in Zhuang ethnic mothers, and provided a reference for maternal and child care administration among the Zhuang population.

\section{MATERIALS AND METHODS}

\subsection{Study Design and Study Population}

This study was approved by the ethical committee of Guangxi Medical University (No. 20140305001) and conducted under the Helsinki Declaration. Informed consent was obtained from all participants.

The present study was conducted as a part of the prospective Guangxi Zhuang Nationality Birth Cohort study which was initiated in 2015. This ongoing cohort enrolls participants at 13 hospitals in the counties of Jingxi, Longan, Tiandong, Tianyang, Pingguo, Wuming, and Debao, which are located in Guangxi Zhuang Autonomous Region in southern China. Pregnant women, who came for their first prenatal care before 12 gestational weeks, were asked to participate in the study. The eligibility criteria for participants are as follows: (1) the pregnant woman and/or her husband are ethnically Zhuang Chinese; (2) residence in the study area; (3) with an expectation to deliver at the collaborating hospital. Participants were invited to complete a questionnaire by a face-to-face interview. The questionnaire used was designed by epidemiologists. A total of 11384 pregnant women were enrolled between June 2015 and July 2018. After excluding those who had multiple births ( $n=549)$, who gave birth to an infant with a birth defect $(n=128)$, and who was not Zhuang ethnic herself ( $n=742)$, a total of 9965 Zhuang ethnic pregnancy women were eventually included in this analysis.

\subsection{Pregnancy Outcomes}

Information about the mothers' disease, history of pregnancy outcomes, and information regarding the infant sex, birth date, birth weight, and gestational age at birth were retrieved from medical records. Gestational age was determined by both the woman's last menstrual period and ultrasound examination. PB was defined as infants born before 37 weeks of gestation ${ }^{[24]}$. LBW and macrosomia were defined as birthweight $<2500 \mathrm{~g}$ and $\geq 4000 \mathrm{~g}$, respectively ${ }^{[9,10]}$. Trained obstetric nurses measured birth weight immediately after delivery using a calibrated electronic scale.

\subsection{Data Collection}

The face-to-face interviews were conducted with the participants at the first antenatal care visit in the hospital by trained investigators. The interview collected a variety of information, including sociodemographic characteristics (maternal age and occupational status), lifestyle variables (alcohol use during pregnancy, smoking during pregnancy, passive smoking, folic acid intake, cooking fuels, and exercise) and the living conditions (natural ventilation of the house, natural lighting of the house and whether the house was decorated in the last five years). Prepregnancy BMI was calculated as weight $(\mathrm{kg}) /$ height $\left(\mathrm{m}^{2}\right)$.

\subsection{Statistical Analysis}

Categorical data were described as the percentage. All risk factorvariables were firstexamined by univariate analysis to assess the importance of each of them on three adverse pregnancy outcomes. A Bonferroni test was used for Post-hoc pairwise comparison. When a variable was found to be significant at the 0.1 level or was reported to be associated with pregnancy outcomes in previous studies, it was entered into the multivariate model.

The correlation between risk factors and adverse pregnancy outcomes was estimated using logistic regression and expressed as odds ratios (OR) and 95\% confidence intervals (CI). A two-tailed value of $P<0.05$ was considered statistically significant. All statistical analyses were performed by IBM SPSS statistics (version 22.0).

\section{RESULTS}

\subsection{Characteristics of Study Population}

A total of 9965 Zhuang ethnic pregnant women were included in the study. The basic characteristics of the pregnant women are shown in table 1 . Women enrolled had a mean age of 28.7 years $(\mathrm{SD}=5.5)$ and were predominantly non-drinkers and nonsmokers. Over half of infants were male. The incidence of PB, LBW, and macrosomia was $5.55 \%, 5.64 \%$ and $2.19 \%$, respectively.

\subsection{Univariate and Multiple Analyses of Factors Related to Adverse Pregnancy Outcomes}

The associations of maternal and fetal factors with the risk of $\mathrm{PB}$ are presented in table 2. In univariate analyses, maternal age $\geq 36$ years was associated with an increased risk of $\mathrm{PB}(\mathrm{OR}=1.43,95 \%$ CI: $1.09-1.88)$, and this association remained statistically significant in 
Table 1 Characteristics of mothers and infants $(n=9965)$

\begin{tabular}{|c|c|}
\hline Characteristics & $n(\%)$ \\
\hline \multicolumn{2}{|l|}{ Maternal age (years) } \\
\hline$\leq 25$ & $2849(28.59)$ \\
\hline $26-30$ & $3438(34.50)$ \\
\hline $31-35$ & $2555(25.64)$ \\
\hline$\geq 36$ & $1123(11.27)$ \\
\hline \multicolumn{2}{|l|}{ Occupational status } \\
\hline Unemployed & $1819(18.25)$ \\
\hline Employed & $8146(81.75)$ \\
\hline \multicolumn{2}{|l|}{ Pre-pregnancy BMI $\left(\mathrm{kg} / \mathrm{m}^{2}\right)$} \\
\hline Underweight $(<18.5)$ & $1464(14.69)$ \\
\hline Normal weight (18.5-24.9) & $4697(47.13)$ \\
\hline Overweight or obesity $(\geq 25)$ & $589(5.91)$ \\
\hline Missing & $3215(32.26)$ \\
\hline \multicolumn{2}{|l|}{ Smoking during pregnancy } \\
\hline No & $9958(99.93)$ \\
\hline Yes & $7(0.07)$ \\
\hline \multicolumn{2}{|l|}{ Passive smoking } \\
\hline No & $5404(54.23)$ \\
\hline Yes & $3905(39.19)$ \\
\hline Missing & $656(6.58)$ \\
\hline \multicolumn{2}{|l|}{ Drinking during pregnancy } \\
\hline No & $9915(99.50)$ \\
\hline Yes & $50(0.50)$ \\
\hline \multicolumn{2}{|l|}{ Folic acid intake } \\
\hline No & $3850(38.64)$ \\
\hline Yes & $6089(61.10)$ \\
\hline Missing & $26(0.26)$ \\
\hline \multicolumn{2}{|l|}{ Cooking fuels } \\
\hline Clean fuels & $8561(85.91)$ \\
\hline Non clean fuels & $1378(13.83)$ \\
\hline Missing & $26(0.26)$ \\
\hline \multicolumn{2}{|l|}{ Natural ventilation of house } \\
\hline Poor or general ventilation & $3103(31.14)$ \\
\hline Good ventilation & $6853(6877)$ \\
\hline Missing & $9(0.09)$ \\
\hline \multicolumn{2}{|l|}{ Natural lighting of house } \\
\hline Poor or general natural lighting & $3148(31.59)$ \\
\hline Good natural lighting & $6808(68.32)$ \\
\hline Missing & $9(0.09)$ \\
\hline \multicolumn{2}{|c|}{ Decorated the house in the last five years } \\
\hline No & $8361(83.90)$ \\
\hline Yes & $1599(16.05)$ \\
\hline Missing & $5(0.05)$ \\
\hline \multicolumn{2}{|l|}{ Regular exercise } \\
\hline No & $9516(95.49)$ \\
\hline Yes & $271(2.72)$ \\
\hline Missing & $178(1.79)$ \\
\hline \multicolumn{2}{|l|}{ Gestational diabetes mellitus } \\
\hline No & $9510(95.44)$ \\
\hline Yes & $455(4.57)$ \\
\hline \multicolumn{2}{|l|}{ Pregnancy induced hypertension } \\
\hline No & $9548(95.82)$ \\
\hline Yes & $417(4.18)$ \\
\hline \multicolumn{2}{|l|}{ Parity } \\
\hline Nulliparous & $5349(53.68)$ \\
\hline Multiparous & $4616(46.32)$ \\
\hline \multicolumn{2}{|l|}{ Infant sex } \\
\hline Male & $5266(52.84)$ \\
\hline Female & $4699(47.16)$ \\
\hline Adverse pregnancy outcomes & \\
\hline $\mathrm{PB}$ & $553(5.55)$ \\
\hline LBW & $562(5.64)$ \\
\hline Macrosomia & $218(2.19)$ \\
\hline
\end{tabular}

$n$, number; PB, preterm birth; LBW, low birth weight; BMI, body mass index multivariate analyses $(\mathrm{OR}=2.22,95 \% \mathrm{CI}$ : 1.51-3.27). No other factors were found to be associated with PB.

Results of univariate and multivariate analyses of factors related to LBW are shown in table 3. In univariate models, those who were underweight $(\mathrm{OR}=1.87,95 \%$ CI: $1.48-2.35)$, with gestational age $<$ 37 weeks (OR=24.58, 95\% CI: 20.08-30.09), and had a female fetus $(\mathrm{OR}=1.53,95 \%$ CI: $1.29-1.82)$ were more likely to have LBW infants, while mothers with age between 31 and 35 years $(\mathrm{OR}=0.63,95 \% \mathrm{CI}: 0.50$ $0.80)$, and multiparous women $(\mathrm{OR}=0.74,95 \% \mathrm{CI}$ : $0.62-0.88$ ) had a lower risk of having a LBW infant. In multivariate models, these associations remained statistically significant except for the association between maternal age and LBW.

The associations of maternal and fetal factors with the risk of macrosomia are presented in table 4. Maternal age between 31 and 35 years $(\mathrm{OR}=1.76$, 95\% CI: $1.03-2.99)$ and pre-pregnancy BMI $\geq 25 \mathrm{~kg} /$ $\mathrm{m}^{2}(\mathrm{OR}=1.79,95 \%$ CI: $1.15-2.80)$ were risk factors for macrosomia; while pre-pregnancy BMI $<18.5 \mathrm{~kg} /$ $\mathrm{m}^{2}(\mathrm{OR}=0.30,95 \% \mathrm{CI}: 0.15-0.60)$ and having a female fetus (OR $=0.41,95 \%$ CI: $0.28-0.59$ ) were protective factors for macrosomia in multivariate analyses. Besides, gestational diabetes mellitus was associated with an increased risk of macrosomia at the brink of significance (OR=1.73, 95\% CI: 0.97-3.09; $P=0.063)$.

\section{DISCUSSION}

In this large population-based study, we examined the incidence of PB, LBW and macrosomia in Zhuang population, and identified the possible risk factors associated with the three adverse pregnancy outcomes. To the best of our knowledge, this is the first cohort study to investigate the incidence and risk factors for adverse pregnancy outcomes in the Zhuang population. The results of the present study suggested that in Zhuang population, maternal age $\geq 36$ years was related to an increased risk of PB; pre-pregnancy underweight, gestational age $<37$ weeks, and having a female fetus were associated with the risk of LBW; maternal age between 31-35 years, pre-pregnancy overweight or obesity, and male fetus might be the risk factors of macrosomia.

In our study, the incidence of $\mathrm{PB}, \mathrm{LBW}$ and macrosomia in Zhuang population was 5.55\%, 5.64\% and $2.19 \%$, respectively. The results of previous studies about the incidence of adverse pregnancy outcomes are shown in table 5, and more than $97 \%$ of the pregnant women were ethnic Han in these studie ${ }^{[18,}$ ${ }^{25-30]}$. The rate of $\mathrm{PB}$ in the present study was similar to that in other studies in China ${ }^{[25-29]}$. The incidence of LBW in our study population was $5.64 \%$, which was higher than the values of studies in Shanghai $(2.9 \%)$, Ma'anshan (1.9\%), Wuhan (4.4\%) and rural areas 
Table 2 Association of maternal and fetal factors with the risk of PB

\begin{tabular}{|c|c|c|c|c|}
\hline \multirow{2}{*}{ Factors } & \multicolumn{2}{|c|}{ Univariate logistic regression } & \multicolumn{2}{|c|}{ Multiple logistic regression ${ }^{\mathrm{a}}$} \\
\hline & OR $(95 \% \mathrm{CI})$ & $P$ & OR $(95 \% \mathrm{CI})$ & $P$ \\
\hline \multicolumn{5}{|l|}{ Maternal age (years) } \\
\hline$\leq 25$ & Reference & & & \\
\hline $26-30$ & $0.88(0.70,1.10)$ & 0.269 & $0.96(0.70,1.31)$ & 0.780 \\
\hline $31-35$ & $1.03(0.82,1.30)$ & 0.797 & $1.24(0.88,1.75)$ & 0.226 \\
\hline$\geq 36$ & $1.43(1.09,1.88)$ & 0.010 & $2.22(1.51,3.27)$ & $<0.001$ \\
\hline \multicolumn{5}{|l|}{ Occupational status } \\
\hline Employed & Reference & & & \\
\hline Unemployed & $0.96(0.77,1.20)$ & 0.739 & & \\
\hline \multicolumn{5}{|l|}{ Pre-pregnancy BMI $\left(\mathrm{kg} / \mathrm{m}^{2}\right)^{\mathrm{b}}$} \\
\hline Underweight $(<18.5)$ & $1.08(0.83,1.40)$ & 0.571 & $1.12(0.85,1.49)$ & 0.410 \\
\hline Normal weight (18.5-24.9) & Reference & & & \\
\hline Overweight or obesity $(\geq 25)$ & $0.93(0.62,1.39)$ & 0.727 & $0.77(0.50,1.19)$ & 0.246 \\
\hline \multicolumn{5}{|l|}{ Passive smoking ${ }^{\mathrm{c}}$} \\
\hline No & Reference & & & \\
\hline Yes & $1.02(0.85,1.22)$ & 0.853 & $1.10(0.87,1.38)$ & 0.420 \\
\hline \multicolumn{5}{|l|}{ Drinking during pregnancy } \\
\hline No & Reference & & & \\
\hline Yes & $0.35(0.05,2.51)$ & 0.294 & $0.53(0.07,3.87)$ & 0.528 \\
\hline \multicolumn{5}{|l|}{ Folic acid intake ${ }^{d}$} \\
\hline No & $1.09(0.92,1.30)$ & 0.333 & $1.10(0.87,1.39)$ & 0.412 \\
\hline Yes & Reference & & & \\
\hline \multicolumn{5}{|l|}{ Cooking fuels ${ }^{\mathrm{e}}$} \\
\hline Clean fuels & Reference & & & \\
\hline Non clean fuels & $1.13(0.89,1.43)$ & 0.335 & $1.18(0.86,1.61)$ & 0.310 \\
\hline \multicolumn{5}{|l|}{ Natural ventilation of house $\mathrm{f}^{\mathrm{f}}$} \\
\hline Good ventilation & Reference & & & \\
\hline Poor or general ventilation & $1.04(0.87,1.26)$ & 0.639 & $1.05(0.83,1.33)$ & 0.692 \\
\hline \multicolumn{5}{|l|}{ Natural lighting of house } \\
\hline Good natural lighting & Reference & & & \\
\hline Poor or general natural lighting & $1.03(0.86-1.24)$ & 0.744 & & \\
\hline \multicolumn{5}{|c|}{ Decorated the house in the last five years ${ }^{\mathrm{g}}$} \\
\hline No & Reference & & & \\
\hline Yes & $1.03(0.82,1.30)$ & 0.791 & $1.13(0.85,1.52)$ & 0.404 \\
\hline \multicolumn{5}{|l|}{ Regular exercise ${ }^{\mathrm{h}}$} \\
\hline No & $1.27(0.71,2.28)$ & 0.423 & $1.66(0.73,3.80)$ & 0.226 \\
\hline Yes & Reference & & & \\
\hline \multicolumn{5}{|l|}{ Gestational diabetes mellitus } \\
\hline No & Reference & & & \\
\hline Yes & $0.90(0.59,1.38)$ & 0.637 & $1.21(0.74,1.97)$ & 0.447 \\
\hline \multicolumn{5}{|l|}{ Pregnancy induced hypertension } \\
\hline No & Reference & & & \\
\hline Yes & $1.14(0.76,1.71)$ & 0.532 & $1.16(0.76,1.77)$ & 0.493 \\
\hline \multicolumn{5}{|l|}{ Parity } \\
\hline Nulliparous & Reference & & & \\
\hline Multiparous & $0.95(0.80,1.12)$ & 0.530 & $0.81(0.63,1.05)$ & 0.109 \\
\hline \multicolumn{5}{|l|}{ Infant sex } \\
\hline Male & Reference & & & \\
\hline Female & $0.89(0.75,1.05)$ & 0.167 & $0.86(0.69,1.08)$ & 0.194 \\
\hline
\end{tabular}

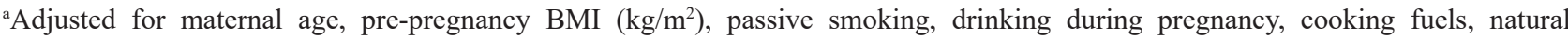
ventilation of house, decorated the house in the last five years, regular exercise, parity, gestational diabetes mellitus, pregnancy induced hypertension, parity, and infant sex.

'Information on pre-pregnancy BMI $\left(\mathrm{kg} / \mathrm{m}^{2}\right)$ missing for 3215 mothers;

'Information on passive smoking missing for 656 mothers;

'Information on folic acid intake missing for 26 mothers;

eInformation on cooking fuels missing for 26 mothers;

fInformation on natural ventilation of house missing for 9 mothers;

Information on having decorated the house in the last five year missing for 5 mothers;

hInformation on regular exercise missing for 178 mothers;

$\mathrm{PB}$, preterm birth; OR, odds ratio; CI, confidence interval; BMI, body mass index 
Table 3 Association of maternal and fetal factors with the risk of LBW

\begin{tabular}{|c|c|c|c|c|}
\hline \multirow{2}{*}{ Factors } & \multicolumn{2}{|c|}{ Univariate logistic regression } & \multicolumn{2}{|c|}{ Multiple logistic regression } \\
\hline & OR $(95 \% \mathrm{CI})$ & $P$ & OR $(95 \% \mathrm{CI})$ & $P$ \\
\hline \multicolumn{5}{|l|}{ Maternal age (years) } \\
\hline$\leq 25$ & Reference & & & \\
\hline $26-30$ & $0.79(0.64,0.97)$ & 0.024 & $1.10(0.80,1.50)$ & 0.557 \\
\hline $31-35$ & $0.63(0.50,0.80)$ & $<0.001$ & $0.87(0.59,1.27)$ & 0.463 \\
\hline$\geq 36$ & $0.88(0.66,1.17)$ & 0.389 & $1.10(0.69,1.74)$ & 0.685 \\
\hline \multicolumn{5}{|l|}{ Occupational status } \\
\hline Employed & Reference & & & \\
\hline Unemployed & $1.02(0.82,1.27)$ & 0.874 & & \\
\hline \multicolumn{5}{|l|}{ Pre-pregnancy BMI $\left(\mathrm{kg} / \mathrm{m}^{2}\right)^{\mathrm{b}}$} \\
\hline Underweight $(<18.5)$ & $1.87(1.48,2.35)$ & $<0.001$ & $1.91(1.45,2.51)$ & $<0.001$ \\
\hline Normal weight (18.5-24.9) & Reference & & & \\
\hline Overweight or obesity $(\geq 25)$ & $0.68(0.43,1.10)$ & 0.120 & $0.76(0.44,1.29)$ & 0.300 \\
\hline \multicolumn{5}{|l|}{ Passive smoking ${ }^{\mathrm{c}}$} \\
\hline No & Reference & & & \\
\hline Yes & $1.08(0.90,1.29)$ & 0.410 & $1.15(0.90,1.48)$ & 0.271 \\
\hline \multicolumn{5}{|l|}{ Drinking during pregnancy } \\
\hline No & Reference & & & \\
\hline Yes & $0.34(0.05,2.47)$ & 0.286 & $0.50(0.06,4.11)$ & 0.522 \\
\hline \multicolumn{5}{|l|}{ Folic acid intake ${ }^{d}$} \\
\hline No & $1.11(0.93,1.32)$ & 0.236 & $1.16(0.90,1.50)$ & 0.240 \\
\hline Yes & Reference & & & \\
\hline \multicolumn{5}{|l|}{ Cooking fuels $\mathrm{e}^{\mathrm{e}}$} \\
\hline Clean fuels & Reference & & & \\
\hline Non clean fuels & $1.20(0.95,1.52)$ & 0.125 & $1.32(0.95,1.84)$ & 0.100 \\
\hline \multicolumn{5}{|l|}{ Natural ventilation of house $\mathrm{f}^{\mathrm{f}}$} \\
\hline Good ventilation & Reference & & & \\
\hline Poor or general ventilation & $1.165(0.97,1.39)$ & 0.095 & $1.26(0.98,1.63)$ & 0.072 \\
\hline \multicolumn{5}{|l|}{ Natural lighting of house } \\
\hline Good natural lighting & Reference & & & \\
\hline Poor or general natural lighting & $1.159(0.97,1.39)$ & 0.106 & & \\
\hline \multicolumn{5}{|c|}{ Decorated the house in the last five years ${ }^{\mathrm{g}}$} \\
\hline No & Reference & & & \\
\hline Yes & $1.22(0.98,1.51)$ & 0.081 & $1.14(0.83,1.56)$ & 0.416 \\
\hline \multicolumn{5}{|l|}{ Regular exercise $^{\mathrm{h}}$} \\
\hline No & $1.56(0.82,2.95)$ & 0.173 & $1.62(0.66,3.97)$ & 0.294 \\
\hline Yes & Reference & & & \\
\hline \multicolumn{5}{|l|}{ Gestational diabetes mellitus } \\
\hline No & Reference & & & \\
\hline Yes & $0.72(0.45,1.15)$ & 0.168 & $0.85(0.45,1.60)$ & 0.610 \\
\hline \multicolumn{5}{|l|}{ Pregnancy induced hypertension } \\
\hline No & Reference & & & \\
\hline Yes & $1.02(0.67,1.56)$ & 0.917 & $0.99(0.61,1.61)$ & 0.971 \\
\hline \multicolumn{5}{|l|}{ Parity } \\
\hline Nulliparous & Reference & & & \\
\hline Multiparous & $0.74(0.62,0.88)$ & $<0.001$ & $0.73(0.55,0.96)$ & 0.024 \\
\hline \multicolumn{5}{|l|}{ Infant sex } \\
\hline Male & Reference & & & \\
\hline Female & $1.53(1.29,1.82)$ & $<0.001$ & $1.74(1.36,2.23)$ & $<0.001$ \\
\hline \multicolumn{5}{|l|}{ Gestational age } \\
\hline$\geq 37$ weeks & Reference & & & \\
\hline$<37$ weeks & $24.58(20.08,30.09)$ & $<0.001$ & $25.77(19.54,33.99)$ & $<0.001$ \\
\hline
\end{tabular}

${ }^{a}$ Adjusted for maternal age, pre-pregnancy BMI $\left(\mathrm{kg} / \mathrm{m}^{2}\right)$, passive smoking, drinking during pregnancy, cooking fuels, natural ventilation of house, decorated the house in the last five years, regular exercise, gestational diabetes mellitus, pregnancy induced hypertension, parity, infant sex, and gestational age.

${ }^{b}$ Information on pre-pregnancy BMI $\left(\mathrm{kg} / \mathrm{m}^{2}\right)$ missing for 3215 mothers;

'Information on passive smoking missing for 656 mothers;

dInformation on folic acid intake missing for 26 mothers;

eInformation on cooking fuels missing for 26 mothers;

Information on natural ventilation of house missing for 9 mothers;

g Information on having decorated the house in the last five year missing for 5 mothers;

hInformation on regular exercise missing for 178 mothers;

LBW, low birth weight; OR, odds ratio; CI, confidence interval; BMI, body mass index. 
Table 4 Association of maternal and fetal factors with the risk of macrosomia

\begin{tabular}{|c|c|c|c|c|}
\hline \multirow{2}{*}{ Factors } & \multicolumn{2}{|c|}{ Univariate logistic regression } & \multicolumn{2}{|c|}{ Multiple logistic regression ${ }^{\mathrm{a}}$} \\
\hline & OR $(95 \% \mathrm{CI})$ & $P$ & OR $(95 \% \mathrm{CI})$ & $P$ \\
\hline \multicolumn{5}{|l|}{ Maternal age (years) } \\
\hline$\leq 25$ & Reference & & & \\
\hline $26-30$ & $1.95(1.27,3.00)$ & 0.002 & $1.30(0.78,2.18)$ & 0.316 \\
\hline $31-35$ & $3.23(2.13,4.92)$ & $<0.001$ & $1.76(1.03,2.99)$ & 0.038 \\
\hline$\geq 36$ & $2.84(1.73,4.69)$ & $<0.001$ & $1.33(0.70,2.54)$ & 0.380 \\
\hline \multicolumn{5}{|l|}{ Occupational status } \\
\hline Employed & Reference & & & \\
\hline Unemployed & $0.94(0.66,1.34)$ & 0.751 & & \\
\hline \multicolumn{5}{|l|}{ Pre-pregnancy BMI $\left(\mathrm{kg} / \mathrm{m}^{2}\right)^{\mathrm{b}}$} \\
\hline Underweight $(<18.5)$ & $0.24(0.12,0.48)$ & $<0.001$ & $0.30(0.15,0.60)$ & 0.001 \\
\hline Normal weight (18.5-24.9) & Reference & & & \\
\hline Overweight or Obesity $(\geq 25)$ & $1.97(1.29,3.01)$ & 0.002 & $1.79(1.15,2.80)$ & 0.010 \\
\hline \multicolumn{5}{|l|}{ Passive smoking ${ }^{\mathrm{c}}$} \\
\hline No & Reference & & & \\
\hline Yes & $0.87(0.65,1.15)$ & 0.316 & $1.00(0.71,1.41)$ & 0.989 \\
\hline \multicolumn{5}{|l|}{ Drinking during pregnancy } \\
\hline No & Reference & & & \\
\hline Yes & $1.87(0.45,7.75)$ & 0.388 & $1.31(0.17,9.92)$ & 0.791 \\
\hline \multicolumn{5}{|l|}{ Folic acid intake ${ }^{d}$} \\
\hline No & $0.97(0.74,1.28)$ & 0.839 & $0.91(0.65,1.29)$ & 0.611 \\
\hline Yes & Reference & & & \\
\hline \multicolumn{5}{|l|}{ Cooking fuels ${ }^{\mathrm{e}}$} \\
\hline Clean fuels & Reference & & & \\
\hline Non-clean fuels & $0.77(0.50,1.19)$ & 0.238 & & \\
\hline \multicolumn{5}{|l|}{ Natural ventilation of house $\mathrm{f}^{\mathrm{f}}$} \\
\hline Good ventilation & Reference & & & \\
\hline Poor or general ventilation & $0.76(0.56,1.03)$ & 0.078 & $0.90(0.63,1.29)$ & 0.560 \\
\hline \multicolumn{5}{|l|}{ Natural lighting of house } \\
\hline Good natural lighting & Reference & & & \\
\hline Poor or general natural lighting & $0.78(0.58,1.06)$ & 0.108 & & \\
\hline \multicolumn{5}{|c|}{ Decorated the house in the last five years } \\
\hline No & Reference & & & \\
\hline Yes & $1.00(0.69,1.44)$ & 1.000 & & \\
\hline \multicolumn{5}{|l|}{ Regular exercise $\mathrm{e}^{\mathrm{g}}$} \\
\hline No & $1.00(0.44,2.28)$ & 0.997 & $1.08(0.39,2.98)$ & 0.882 \\
\hline Yes & Reference & & & \\
\hline \multicolumn{5}{|l|}{ Gestational diabetes mellitus } \\
\hline No & Reference & & & \\
\hline Yes & $2.16(1.35,3.46)$ & 0.001 & $1.73(0.97,3.09)$ & 0.063 \\
\hline \multicolumn{5}{|l|}{ Pregnancy induced hypertension } \\
\hline No & Reference & & & \\
\hline Yes & $0.87(0.43,1.77)$ & 0.701 & $0.76(0.37,1.57)$ & 0.460 \\
\hline \multicolumn{5}{|l|}{ Parity } \\
\hline Nulliparous & Reference & & & \\
\hline Multiparous & $1.51(1.16,1.98)$ & 0.003 & $1.30(0.88,1.93)$ & 0.192 \\
\hline \multicolumn{5}{|l|}{ Infant sex } \\
\hline Male & Reference & & & \\
\hline Female & $0.45(0.33,0.60)$ & $<0.001$ & $0.41(0.28,0.59)$ & $<0.001$ \\
\hline
\end{tabular}

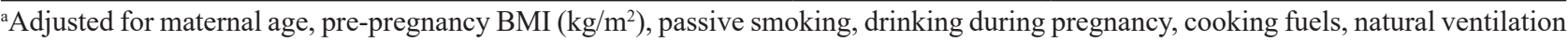
of house, regular exercise, gestational diabetes mellitus, pregnancy induced hypertension, parity, and infant sex.

${ }^{b}$ Information on pre-pregnancy BMI $\left(\mathrm{kg} / \mathrm{m}^{2}\right)$ missing for 3215 mothers;

'Information on passive smoking missing for 656 mothers;

'Information on folic acid intake missing for 26 mothers;

eInformation on cooking fuels missing for 26 mothers;

fInformation on natural ventilation of house missing for 9 mothers;

Information on regular exercise missing for 178 mothers;

LBW, low birth weight; OR, odds ratio; CI, confidence interval; BMI, body mass index. 
Table 5 Comparison of the incidence of adverse pregnancy outcomes in the present study and other studies in China

\begin{tabular}{lccccccc}
\hline Locations & Urban/Rural & Year & Total & PB (\%) & LBW (\%) & Macrosomia (\%) & Reference \\
\hline Guangxi & Rural & $2015-2018$ & 9965 & 5.55 & 5.64 & 2.19 & Present study \\
Shanghai & Urban & $2013-2016$ & 4015 & 5.6 & 2.9 & 8.2 & {$[25]$} \\
Ma'anshan, Anhui & Urban\&Rural & $2013-2014$ & 3079 & 3.2 & 1.9 & 6.9 & {$[26]$} \\
Wuhan, Hubei & Urban & $2012-2014$ & 10111 & 6.4 & 4.4 & - & {$[27]$} \\
Guangzhou, Guangdong & Urban & $2012-2016$ & 14093 & 6.1 & 6.7 & - & {$[28]$} \\
A nationwide study & Rural & $2010-2012$ & 367425 & 5.21 & - & - & {$[29]$} \\
Shaanxi & Rural & $2010-2013$ & 809 & - & 3.6 & - & {$[30]$} \\
Xi'an, Shaanxi & Rural & $2010-2013$ & 181 & - & - & 8.6 & {$[18]$} \\
\hline
\end{tabular}

of Shaanxi province $(3.6 \%)$, but lower than that in Guangzhou $(6.7 \%)^{[25-28,30]}$. Compared to the previous studies, the Zhuang population in our study had a lower macrosomia incidence $(8.2 \%$ in Shanghai, $6.9 \%$ in Ma' anshan, and $8.6 \%$ in rural areas of Xi' an $)^{[18,25,26]}$.

It has been demonstrated that maternal prepregnancy BMI was associated with the infant birth weight: women with low pre-pregnancy BMI had a higher risk of giving birth to LBW babies; on the other hand, high pre-pregnancy BMI was a risk factor for macrosomia ${ }^{[20,31,32]}$. The Zhuang women in our study had lower pre-pregnancy BMI $(5.91 \%$ overweight or obesity) than women in other cohort study $(9 \%$ overweight or obesity in Shanghai and $11.72 \%$ in Ma'anshan) ${ }^{[26,33]}$. Thus, the higher LBW incidence and the lower macrosomia incidence of Zhuang fetal may be related to the lower pre-pregnancy BMI of their mothers. Furthermore, some researchers suggested that maternal and child health outcomes may vary by health inequalities between different ethnic groups ${ }^{[34]}$. The factors contributing to health inequalities between ethnic and non-ethnic minority populations are varied and complex, while some studies suggested that the income, education, employment, social policies, health care systems, health behaviors, cultural norms, and maternal and child health service coverage may all play a role ${ }^{[34-36]}$.

Our study showed that mothers with maternal age $\geq 36$ years were more likely to deliver preterm babies. It is consistent with the previous studies ${ }^{[37,38]}$. Previous evidence showed that the risk of pregnancy outcome would be greater in advanced age due to placental physiological changes of aging ${ }^{[37,39]}$. It should be noted that the average age of childbearing in China has increased from 26.31 in 2000 to 29.13 in $2010^{[38]}$. Therefore, clinical workers should strengthen the prevention and health care of pregnant women in advanced maternal age to avoid serious adverse pregnancy outcomes.

LBW remains an important public health issue for developing countries ${ }^{[40]}$. Our study demonstrated that pre-pregnancy BMI $<18.5 \mathrm{~kg} / \mathrm{m}^{2}$, nulliparous, and female fetus were risk factors for LBW. The possible mechanism is as follows. Low pre-pregnancy weight women may not be able to provide adequate nutrients to the fetus, ultimately leading to $\mathrm{LBW}^{[41]}$. Increasing parity is related to a progressive increase in uterine blood flow, which leads to increased birth weight of subsequent offspring ${ }^{[15]}$. In terms of infant sex, the weight difference between male and female infants may be associated with chromosomes and hormones ${ }^{[42]}$.

Maternal age between 31-35 years old and prepregnancy overweight or obesity were related to a higher risk of macrosomia, which is consistent with several other studies ${ }^{[43,44]}$. Both two factors can lead to macrosomia by affecting fetal fat and protein stores ${ }^{[43,44]}$. We also found that male fetuses were more likely to be macrosomia. This is consistent with differences in fetal growth between males and females ${ }^{[42]}$. In addition, we found that gestational diabetes mellitus tended to be a risk factor for macrosomia. The excessive shunting of nutrients to the fetus and the acceleration of fetal growth trajectory may contribute to the increased risk of macrosomia in women with gestational diabetes mellitus ${ }^{[45]}$.

One of the strengths of our study is that the prospective cohort design was used. The information was collected in the early stages of pregnancy, which can reduce the recall bias. In addition, interviews conducted with all participants provided the opportunity to adjust for more potential risk factors for adverse pregnancy outcomes, such as maternal pre-pregnancy BMI, regular exercise, passive smoking, and cooking fuels. However, the study has some limitations. First, some variables have missing data, e.g. pre-pregnancy BMI, passive smoking, natural ventilation of house, and natural lighting of house. Second, maternal nutritional status was not addressed in this study. It is common knowledge that low maternal levels of nutrients are related to adverse effects on fetal development.

In conclusion, the incidence of $\mathrm{PB}, \mathrm{LBW}$ and macrosomia in Zhuang people was 5.5\%, 5.64\% and $2.19 \%$, respectively. In Zhuang pregnant women, maternal age $\geq 36$ years was related to a higher incidence of PB. Mothers who were pre-pregnancy underweight, with gestational age $<37$ weeks, and had a female fetus were more likely to have LBW infants. Maternal age between 31-35 years, pre-pregnancy overweight or obesity, and male fetus were linked to increased risk of macrosomia. Health service at the perinatal stage 
should be strengthened for pregnant women with one of these risk factors to reduce the incidence of adverse pregnancy outcomes. The potential mechanism of these risk factors should be studied in future studies.

\section{Acknowledgments}

We are grateful to the pregnant women in the study, the research team from School of Public Health at Guangxi Medical University, and the medical staffs of 13 hospitals, including Jingxi People's Hospital, Jingxi Maternity and Child Health Care Hospital, Longan People's Hospital, Tiandong People's Hospital, Tiandong Maternity and Child Health Care Hospital, Tianyang People's Hospital, Tianyang Maternity and Child Health Care Hospital, Pingguo People's Hospital, Pingguo Maternity and Child Health Care Hospital, Debao People's Hospital, Debao Maternity and Child Health Care Hospital, Wuming People's Hospital, and Wuming Maternity and Child Health Care Hospital.

\section{Open Access}

This article is licensed under a Creative Commons Attribution 4.0 International License https://creativecommons.org/licenses/by/4.0/), which permits use, sharing, adaptation, distribution and reproduction in any medium or format, as long as you give appropriate credit to the original author(s) and the source, provide a link to the Creative Commons licence, and indicate if changes were made. The images or other third party material in this article are included in the article's Creative Commons licence, unless indicated otherwise in a credit line to the material. If material is not included in the article's Creative Commons licence and your intended use is not permitted by statutory regulation or exceeds the permitted use, you will need to obtain permission directly from the copyright holder. To view a copy of this licence, visit http://creativecommons. org/licenses/by/4.0/.

\section{Conflict of Interest Statement}

The authors report no conflicts of interest in this study.

\section{REFERENCES}

1 Blencowe $\mathrm{H}$, Cousens S, Oestergaard MZ, et al. National, regional, and worldwide estimates of preterm birth rates in the year 2010 with time trends since 1990 for selected countries: a systematic analysis and implications. Lancet, 2012,379(9832):2162-2172

2 Vogel JP, Chawanpaiboon S, Moller AB, et al. The global epidemiology of preterm birth. Best Pract Res Clin Obstet Gynaecol, 2018,52:3-12

3 Evensen KA, Steinshamn S, Tjonna AE, et al. Effects of preterm birth and fetal growth retardation on cardiovascular risk factors in young adulthood. Early Hum Dev, 2009,85(4):239-245

4 Crump C, Sundquist J, Winkleby MA, et al. Gestational age at birth and mortality from infancy into midadulthood: a national cohort study. Lancet Child Adolesc Health, 2019,3(6):408-417

5 Li S, Xi B. Preterm birth is associated with risk of essential hypertension in later life. Int J Cardiol, 2014, 172(2):e361-363

6 Malacova E, Regan A, Nassar N, et al. Risk of stillbirth, preterm delivery, and fetal growth restriction following exposure in a previous birth: systematic review and meta-analysis. BJOG, 2018,125(2):183-192

7 Huang A, Jin X, Liu X, et al. A matched case-control study of preterm birth in one hospital in Beijing, China. Reprod Health, 2015,12:1

8 Simmons LE, Rubens CE, Darmstadt GL, et al. Preventing preterm birth and neonatal mortality: exploring the epidemiology, causes, and interventions. Semin Perinatol, 2010,34(6):408-415

9 Blencowe H, Krasevec J, de Onis M, et al. National, regional, and worldwide estimates of low birth weight in 2015, with trends from 2000: a systematic analysis. Lancet Glob Health, 2019,7(7):e849-e860

10 Ye JF, Torloni MR, Ota E, et al. Searching for the definition of macrosomia through an outcome-based approach in low- and middle-income countries: a secondary analysis of the WHO Global Survey in Africa, Asia and Latin America. BMC Pregnancy Childbirth, 2015,15:324

11 Liang X, Xiao L, Luo Y, et al. Prevalence and Risk Factors of Childhood Hypertension in Urban-Rural Areas of China: A Cross-Sectional Study. Int J Hypertens, 2020(5):1-18

12 Pan XF, Tang L, Lee AH, et al. Association between fetal macrosomia and risk of obesity in children under 3 years in Western China: a cohort study. World J Pediatr, 2019,15(2):153-160

13 Chaman R, Amiri M, Raei M, et al. Low birth weight and its related risk factors in northeast iran. Iran $\mathrm{J}$ Pediatr, 2013,23(6):701-704

14 Phung H, Bauman A, Nguyen TV, et al. Risk factors for low birth weight in a socio-economically disadvantaged population: parity, marital status, ethnicity and cigarette smoking. Eur J Epidemiol, 2003,18(3):235-243

15 Shah PS. Parity and low birth weight and preterm birth: a systematic review and meta-analyses. Acta Obstet Gynecol Scand, 2010,89(7):862-875

16 Wang J, Zeng Y, Ni ZM, et al. Risk Factors for Low Birth Weight and Preterm Birth: A Population-based Case-control Study in Wuhan, China. J Huazhong Univ Sci Technol [Med Sci], 2017,37(2):286-292

17 Shan X, Chen F, Wang W, et al. Secular trends of low birthweight and macrosomia and related maternal factors in Beijing, China: a longitudinal trend analysis. BMC Pregnancy Childbirth, 2014,14(1):105

18 Zhang Q, Bai RH, Wang LL, et al. Incidence of fetal macrosomia among single live birth neonates and influencing factors in Xi'an, 2010-2013. Zhonghua Liuxingbingxue Zazhi (Chinese), 2016,37(8):1095-1098

19 Lei F, Zhang L, Shen Y, et al. Association between parity and macrosomia in Shaanxi Province of Northwest China. Ital J Pediatr, 2020,46(1):24

20 Vinturache AE, Chaput KH, Tough SC. Pre-pregnancy body mass index (BMI) and macrosomia in a Canadian birth cohort. J Matern Fetal Neonatal Med, 2017,30(1): 109-116

21 Koyanagi A, Zhang J, Dagvadorj A, et al. Macrosomia in 23 developing countries: an analysis of a multicountry, facility-based, cross-sectional survey. Lancet, 2013, 381(9865):476-483

22 Diabelková J, Rimárová K, Urdzík P, et al. Risk factors 
of preterm birth and low birth weight neonates among roma and non-roma mothers. Cent Eur J Public Health, 2018,26(Suppl):S25-S31

23 Zheng S, Chang W, Lin WY, et al. An analysis of status of birth weight among different ethnic groups in Qapqal. Zhongguo Fuyou Baojian (Chinese), 2004,19(23):86-87

24 Spong CY. Defining "term" pregnancy: recommendations from the Defining "Term" Pregnancy Workgroup. JAMA, 2013,309(23):2445-2446

25 Zhang J, Tian Y, Wang W, et al. Cohort profile: The Shanghai Birth Cohort. Int J Epidemiol, 2019(1):21$21 \mathrm{G}$

26 Ge X, Tao Fb, Huang K, et al. Maternal Snoring May Predict Adverse Pregnancy Outcomes: A Cohort Study in China. PLoS One, 2016,11(2):e0148732

27 Song L, Shen L, Li H, et al. Afternoon napping during pregnancy and low birth weight: the Healthy Baby Cohort study. Sleep Med Clin, 2018,48:35-41

28 Qiu X, Lu JH, He JR, et al. The Born in Guangzhou Cohort Study (BIGCS). Eur J Epidemiol, 2017,32(4): 337-346

29 Yang Y, He Y, Li Q, et al. Preconception blood pressure and risk of preterm birth: a large historical cohort study in a Chinese rural population. Fertil Steril, 2015,104(1): 124-130

30 Liu AP, Zhang R, Li ZP, et al. Incidence of low birth weight among single live birth neonates and influencing factors in Shaanxi. Zhonghua Liuxingbingxue Zazhi (Chinese), 2015,36(11):1244-1248

31 Argerich MI, David RA, Bocchini A. GDM-Impact of BMI in Maternal Bioquimical-Metabolic Variables and Its Association with Macrosomia. Diabetes, 2018, 67(Supplement 1):2347-PUB

32 Liu P, Xu L, Wang Y, et al. Association between perinatal outcomes and maternal pre-pregnancy body mass index. Obes Rev, 2016,17(11):1091-1102

33 Wang X, Wang X, Chen Q, et al. Urinary bisphenol a concentration and gestational diabetes mellitus in chinese women. Epidemiology, 2017,28:S41-S47

34 Anderson I, Robson B, Connolly M, et al. Indigenous and tribal peoples' health (The Lancet-Lowitja Institute Global Collaboration): a population study. Lancet, 2016,
388:131-157

35 Stephens C, Nettleton C, Porter J, et al. Indigenous peoples' health-why are they behind everyone, everywhere? Lancet, 2005,366:10-11

36 Huang Y, Shallcross D, Pi L, et al. Ethnicity and maternal and child health outcomes and service coverage in western china: a systematic review and meta-analysis. Lancet Glob Health, 2018,6(1):e39-e56

37 Wong-Taylor LA, Lawrence A, Cowen S, et al. Maternal and neonatal outcomes of spontaneously conceived pregnancies in mothers over 45 years: a review of the literature. Arch Gynecol Obstet, 2012,285(4):11611166

38 Wang C, Wang XY, Yang HX. Effect of maternal age on pregnancy outcomes in Beijing. Zhonghua Fuchanke Zazhi (Chinese), 2017,52(8):514-520

39 Fitzpatrick KE, Tuffnell D, Kurinczuk JJ, et al. Pregnancy at very advanced maternal age: a UK population-based cohort study. BJOG, 2017,124(7): 1097-1106

40 Khan JR, Islam MM, Awan N, et al. Analysis of low birth weight and its co-variants in Bangladesh based on a sub-sample from nationally representative survey. BMC Pediatr, 2018,18(1):100

41 Neggers Y, Goldenberg RL. Some thoughts on body mass index, micronutrient intakes and pregnancy outcome. J Nutr, 2003,133(5 suppl):1737S-1740S

42 Cogswell ME, Yip R. The influence of fetal and maternal factors on the distribution of birthweight. Semin Perinatol, 1995,19(3):222-240

43 Kc K, Shakya S, Zhang H. Gestational diabetes mellitus and macrosomia: a literature review. Ann Nutr Metab, 2015,66 Suppl 2(2):14-20

44 Kenny LC, Lavender T, McNamee R, et al. Advanced maternal age and adverse pregnancy outcome: evidence from a large contemporary cohort. PLoS One, 2013,8(2): e56583

45 Chiefari E, Arcidiacono B, Foti D, et al. Gestational diabetes mellitus: an updated overview. J Endocrinol Invest, 2017,40(9):899-909

(Received Dec. 15, 2020; accepted Feb. 22, 2021) 